\title{
Cranial Arachnoid Protrusions and Contiguous Diploic Veins in CSF Drainage
}

\author{
S. Tsutsumi, I. Ogino, M. Miyajima, M. Nakamura, Y. Yasumoto, H. Arai, and M. Ito
}

\begin{abstract}
BACKGROUND AND PURPOSE: Studies have suggested that arachnoid villi or granulations found in the walls of the cranial dural sinuses, olfactory mucosa, and cranial nerve sheaths function as outlets for intracranial CSF. However, their role as CSF outlets has not yet been verified. Here we show that arachnoid protrusions and contiguous diploic veins provide an alternative drainage route for intracranial CSF.
\end{abstract}

MATERIALS AND METHODS: Four hundred patients with intact skull, dura mater, and dural sinuses underwent MR imaging to explore arachnoids protruding into the skull and diploic veins. Patients with symptoms of increased intracranial pressure or intracranial hypotension were excluded. For 15 patients undergoing craniotomy, both peripheral and diploic venous blood was collected. Albumin and the CSF-specific biomarkers were measured by enzyme-linked immunosorbent assay.

RESULTS: With MR imaging, arachnoid protrusions into the skull and contiguous diploic veins were consistently identified throughout the cranium with their characteristic appearance depending on the cranial region. In addition, elevated amounts of prostaglandin $D$ synthase and cystatin $\mathrm{C}$ were confirmed in diploic veins compared with peripheral venous blood.

CONCLUSIONS: Diploic veins are distributed ubiquitously throughout the cranium. A portion of the intracranial CSF may be drained through arachnoid protrusions and contiguous diploic veins.

ABBREVIATIONS: $A P=$ arachnoid protrusion; $C y s C=$ cystatin $C ; D V=$ diploic vein; $P G D S=$ prostaglandin $D$ synthase

t has been suggested that arachnoid villi or granulations found in the walls of the cranial dural sinuses, olfactory mucosa, and cranial nerve sheaths function as outlets for intracranial CSF. ${ }^{1-4}$ Arachnoid granulations located adjacent to or in the cranial dural sinuses have been explored by using a variety of methods, including neuroimaging, ${ }^{5-15}$ postmortem dissection, ${ }^{5,16,17}$ investigations by using casting material, ${ }^{18}$ and ex vivo studies. ${ }^{1}$ However, no report has actually shown the process of CSF absorption in vivo. Diploic veins (DVs) are present throughout the cranium; however, their distribution and functional implications have rarely been documented. ${ }^{1,19}$ One previous MR imaging study proposed the hypothesis that major pathways of the DVs function as CSF drainage routes. ${ }^{19}$

Lipocalin-type prostaglandin D synthase (PGDS) is a major

Received December 17, 2013; accepted after revision January 11, 2014.

From the Department of Neurological Surgery (S.T., Y.Y., M.I.), Juntendo University Urayasu Hospital, Chiba, Japan; Department of Neurological Surgery (I.O., M.M., H.A.), Juntendo University School of Medicine, Tokyo, Japan; and Division of Radiological Technology (M.N.), Medical Satellite Yaesu Clinic, Tokyo, Japan.

Please address correspondence to Satoshi Tsutsumi, MD, Department of Neurological Surgery, Juntendo University Urayasu Hospital, 2-1-1 Tomioka, Urayasu,

Chiba 279-0021, Japan; e-mail address: shotaro@juntendo-urayasu.jp

http://dx.doi.org/10.3174/ajnr.A4007 endogenous $\beta$ chaperone that is present in the brain and secreted into the CSF. ${ }^{20,21}$ It is thought to be a CSF-specific biomarker and sensitive to inflammatory demyelinating disease, ${ }^{22}$ Alzheimer disease, ${ }^{23}$ and normal pressure hydrocephalus. ${ }^{19}$ Cystatin $\mathrm{C}(\mathrm{CysC})$, a low-molecular-weight cysteine proteinase inhibitor, is known to be expressed in greater amounts in CSF compared with plasma. ${ }^{24}$ The purpose of the present study was to demonstrate that arachnoid pouches protruding into the skull (APs) and contiguous diploic veins provide an alternative CSF drainage route by highresolution MR imaging; and we also aimed to measure CSF-specific biomarkers, PGDS, and CysC.

\section{MATERIALS AND METHODS}

These studies were performed in accordance with the guidelines for human research of our institution. Written informed consent was obtained for all imaging and laboratory studies.

\section{Examinations with MR Imaging and Postmortem Dissection}

All neuroimaging examinations were performed with a 3T MR imaging scanner equipped with a 32-channel head coil. Four hundred patients (mean age, 52 years) with intact skull, dura mater, and dural sinuses were selected as subjects. Patients with symp- 
toms of increased intracranial pressure or intracranial hypotension were excluded. A coronal T2-weighted sequence involving the whole cranial vault was performed in 150 patients to record all the APs in the cranial cavity, with parameters as follows: TR, $5598.69 \mathrm{~ms}$; TE, $100.00 \mathrm{~ms}$; section thickness, $1.00 \mathrm{~mm}$; intersection gap, $0.2 \mathrm{~mm}$; matrix, $230 \times 230$; FOV, $250 \mathrm{~mm}$; flip angle, $90^{\circ}$; and scan duration, 6 minutes 40 seconds. The remaining 250 patients were analyzed with a coronal T2-weighted sequence focusing on the pterional region, with parameters as follows: TR, $4038.35 \mathrm{~ms}$; TE, $90.00 \mathrm{~ms}$; section thickness, $2 \mathrm{~mm}$; intersection gap, $0.2 \mathrm{~mm}$; matrix, $300 \times 189$; FOV , $150 \mathrm{~mm}$; flip angle, $90^{\circ}$; and scan duration, 4 minutes 25 seconds. A contrast study with intravenous gadolinium infusion $(0.1 \mathrm{mmol} / \mathrm{kg})$, involving the whole cranial vault, was performed in 100 of 400 patients with parameters as follows: TR, $4.1 \mathrm{~ms}$; TE, $1.92 \mathrm{~ms}$; section thickness, $1 \mathrm{~mm}$; intersection gap, $0 \mathrm{~mm}$; matrix, $320 \times$ 320; FOV, $250 \mathrm{~mm}$; flip angle, $13^{\circ}$; and scan duration, $4 \mathrm{~min}-$ utes 25 seconds. Imaging data were analyzed independently by 2 authors (Y.Y. and M.I.).

APs piercing the dura mater were also microscopically observed in an injected postmortem head.

\section{Biochemical Analysis}

For 15 patients ( 6 men and 9 women; mean age, 50 years) undergoing craniotomy, both peripheral and diploic venous blood was simultaneously collected. In each patient, $5 \mathrm{~mL}$ of diploic venous blood draining from the bony edges was manually aspirated by using syringes following removal of the bone flap. In 5 patients, CSF was also obtained intraoperatively. All samples of blood and CSF were poured into test tubes without any mounting reagent and were immediately centrifuged to remove cells and debris, aliquoted, and finally stored in polypropylene tubes at $-80^{\circ} \mathrm{C}$ until ready for biochemical analysis. Albumin, PGDS, and CysC were measured in these 15 patients by enzyme-linked immunosorbent assay. Albumin was measured as a reference marker with a larger molecular weight than PGDS and CysC. Results were analyzed by using the Wilcoxon signed rank test (SPSS 18.0 software package; IBM, Armonk, New York). A $P$ value $<.05$ was considered statistically significant.

\section{RESULTS}

On MR imaging, APs were roughly classifiable into vesicular, tubular, and extensive types (Fig $1 B,-C$ ). In the cerebral convexity, APs were commonly identified in the parasagittal region, connecting to either a linear or branching architecture with variable thickness, but showing a consistent course in the diploe. These APs were consistently distributed throughout the pterional region (Fig 1D), middle cranial fossa (Fig $1 E$ ), and posterior cranial fossa (Fig $1 F$ ), with contiguous DVs showing a characteristic course in each cranial region. DVs appeared with intensity similar to that of the CSF on noncontrast sequence imaging, whereas they showed configurations identical to those delineated on contrast-enhanced images. Therefore, we have concluded that both methods visualize the same DVs (Fig $1 G,-H$ ). This conclusion also indicates that DVs can appear both as fluid-filled channels and venous structures on MR imaging.

In 150 patients, the total number of AP-DV pairings located in the cerebral convexity was $987-489$ on the right and 498 on the left (Table 1). When the cerebral convexity was divided into 3 parts (anterior, middle, and posterior third; Fig $1 A$ ), 55\% of the 987 pairings were found in middle, while $27 \%$ were identified in the posterior and $18 \%$ were in the anterior part (Table 1 ). The mean number of AP-DV pairings in 1 subject was 1.2 (range, $0-5$ ) in the anterior part, 3.6 (range, 0-10) in the middle part, and 1.7 (range, $0-8$ ) in the posterior part. In total, an average of 6.6 (range, 0-17) pairings was found in each subject, with an average of 6.9 in the population of subjects older than 18 years of age and 2.3 in the population younger than 18 years. In the cerebral convexity, vesicular APs were most predominant and comprised $68 \%$ of 987 pairings, followed by tubular (22\%) and extensive (10\%) AP types (Fig $1 B)$.

The total number of AP-DV pairings distributed in the skull base was 375 in our 150 patients, 159 on the right and 216 on the left (Table 2). Fifty-three percent of the 375 pairings were located in the middle cranial fossa, and $47 \%$ were located in the posterior fossa. Neither APs nor DV s were identified in the anterior fossa. In the middle cranial fossa, AP-DV pairings were twice as often identified on the left side as on the right. The mean number of pairings in 1 subject was 1.3 (range, $0-8$ ) in the middle cranial fossa and 1.2 (range, $0-7$ ) in the posterior fossa. In total, an average of 2.5 (range, $0-11$ ) pairings were found in each subject, with an average of 2.6 in the population of subjects older than 18 years of age and 0.6 in the population younger than 18 years of age. In the skull base, tubular APs were most predominant and comprised $61 \%$ of pairings in the middle cranial fossa and $94 \%$ in the posterior fossa, followed by vesicular AP pairings. Extensive APs were not found in the skull base (Table 2). In 100\% of the subjects studied, at least 1 arachnoid granulation was found in the superior sagittal sinus, whereas in the transverse sinus, at least 1 was identified in $23 \%$ of subjects on the right and in $37 \%$ of subjects on the left.

In the pterional region, AP-DV pairings were identified in $20 \%$ of 250 patients on the right and in $29 \%$ of patients on the left. The identification of these pairings did not depend on patient age. All of the identified APs were of the tubular type and were located at the lower surface of the lateral lesser sphenoid wing.

In 15 patients who underwent craniotomy in various regions, albumin (66 kDa), PGDS (19 kDa), and CysC (13 kDa) levels were measured in the diploic and peripheral venous blood. In all patients, diploic venous blood was collected with ease. In 5 patients, CSF was also obtained intraoperatively. PGDS and CysC levels in the CSF were $8278.2 \pm 1853.4 \mathrm{ng} / \mathrm{mL}$ and $2044.6 \pm 390.5 \mu \mathrm{g} / \mathrm{L}$, respectively. The PGDS and CysC levels in peripheral venous blood were $107.9 \pm 59.3 \mathrm{ng} / \mathrm{mL}$ and $237.9 \pm 77.8 \mu \mathrm{g} / \mathrm{L}$ and $212.4 \pm 199.8 \mathrm{ng} / \mathrm{mL}$ and $294.9 \pm 94.7$ $\mu \mathrm{g} / \mathrm{L}$, respectively. In these 15 patients, the diploic vein/peripheral vein ratios were significantly elevated for PGDS and CysC, with values of $2.43 \pm 1.99(P=.011)$ and $1.31 \pm 0.43$ $(P=.023)$, respectively (Fig 3$)$.

On microscopic inspection of the cranial cavity, APs penetrated the dura mater through either a single, oval, or slit-like defect (Fig $2 A$ ) or through mesh-like lacunae (Fig 2B). These types of openings were observed throughout the cranial dura mater, 

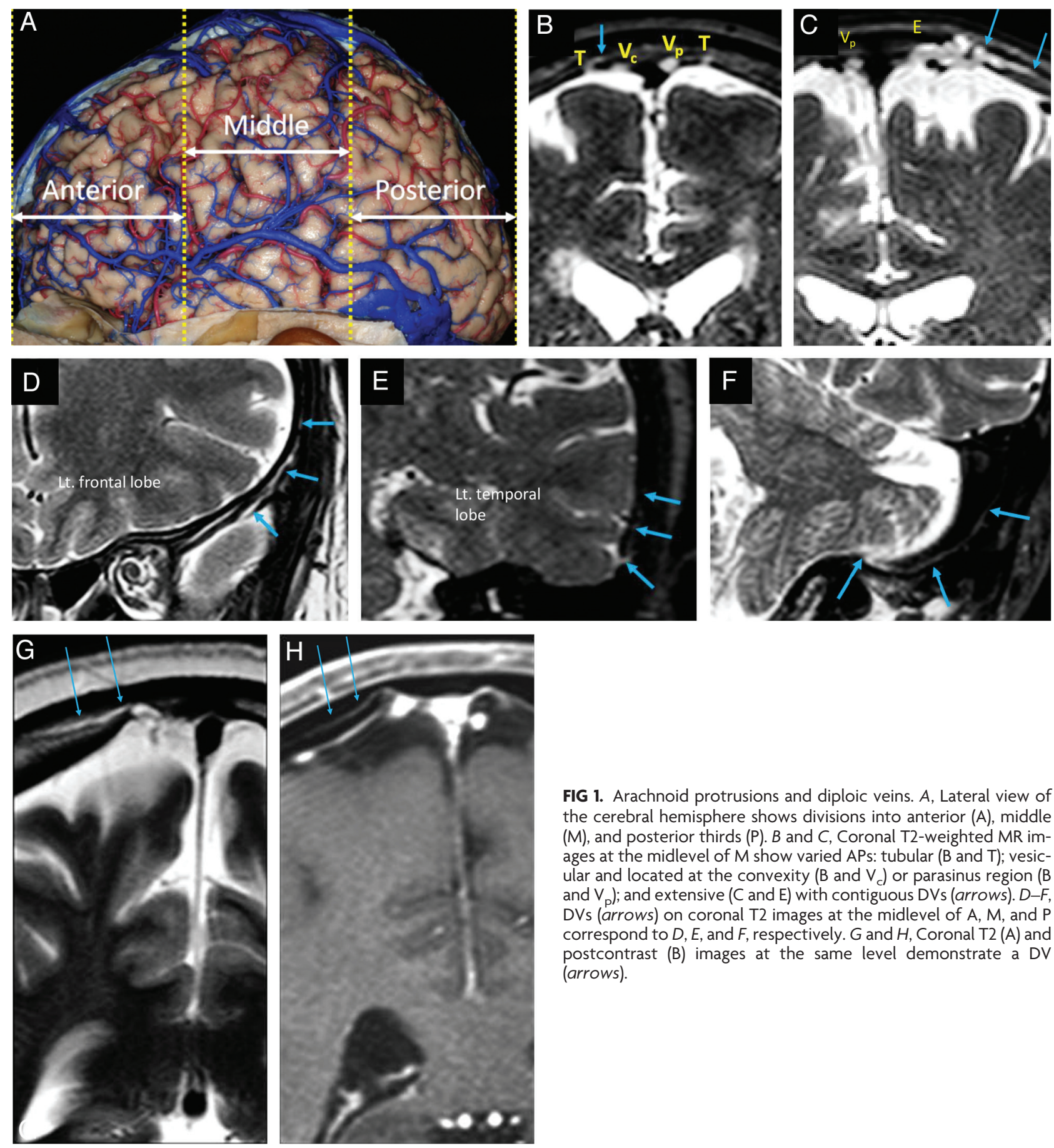

Table 1: Location of AP-DV pairings in the cerebral convexity

\begin{tabular}{lcrc}
\hline & Right & Left & Total \\
\hline A & 98 & 77 & $175(18 \%)$ \\
M & 257 & 288 & $545(55 \%)$ \\
P & 134 & 133 & $267(27 \%)$ \\
& 489 & 498 & 987 \\
\hline
\end{tabular}

Note:-A indicates anterior third; $M$, middle third; $P$, posterior third.

especially in the parasagittal region, and, compared with observations during actual surgeries, a clearer view was obtained in postmortem specimens in which bleeds resulting from disruption of vasculature connecting the dura mater and inner surface of the skull were less likely to disturb anatomic observations.

FIG 1. Arachnoid protrusions and diploic veins. A, Lateral view of the cerebral hemisphere shows divisions into anterior (A), middle $(M)$, and posterior thirds (P). B and C, Coronal T2-weighted MR images at the midlevel of $M$ show varied APs: tubular ( $B$ and T); vesicular and located at the convexity $\left(B\right.$ and $V_{c}$ ) or parasinus region ( $B$ and $\mathrm{V}_{\mathrm{p}}$ ); and extensive ( $\mathrm{C}$ and $\mathrm{E}$ ) with contiguous DVs (arrows). D $-F$, DVs (arrows) on coronal T2 images at the midlevel of $\mathrm{A}, \mathrm{M}$, and $\mathrm{P}$ correspond to $D, E$, and $F$, respectively. $G$ and $H$, Coronal $\mathrm{T} 2(\mathrm{~A})$ and postcontrast (B) images at the same level demonstrate a DV (arrows).

Table 2: Number of AP-DV pairings in the skull base

\begin{tabular}{lccc}
\hline & AF & MF & PF \\
\hline Right & 0 & 69 & 90 \\
Left & 0 & 130 & 86 \\
Tubular & 0 & $121(61 \%)$ & $165(94 \%)$ \\
Vesicular & 0 & $78(39 \%)$ & $11(6 \%)$ \\
Extensive & 0 & 0 & 0 \\
\hline
\end{tabular}

Note:-AF indicates anterior fossa; MF, middle fossa; PF, posterior fossa.

\section{DISCUSSION}

Despite their normal venous structure, DVs are inconsistently visualized by conventional neuroimaging techniques, including digital subtraction angiography. ${ }^{25}$ They have been infrequently associated with dural arteriovenous fistulas, ${ }^{26-28}$ tumorous pa- 

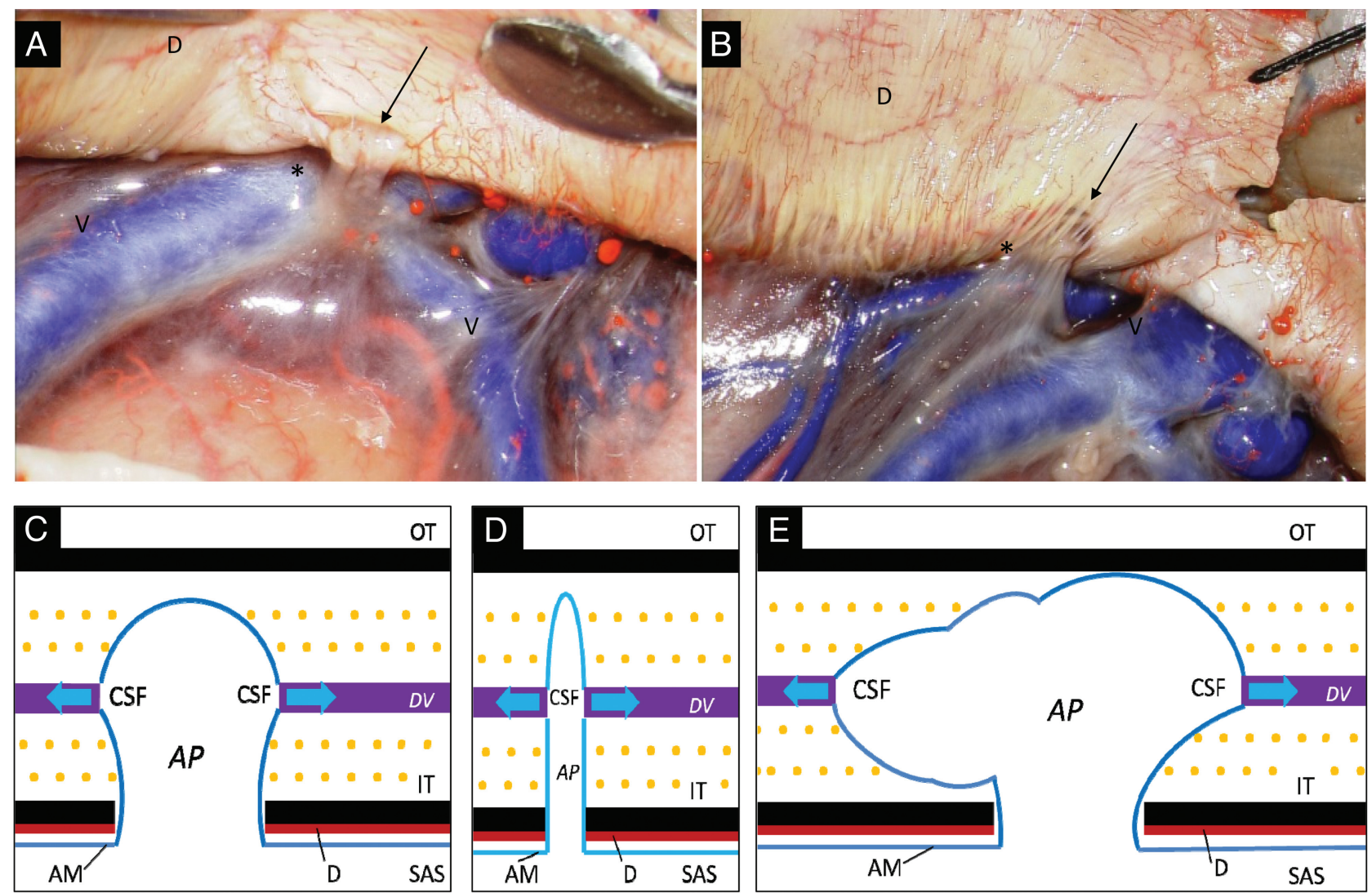

FIG 2. Gross appearance and schema of arachnoid protrusions and contiguous DVs. $A$ and $B$, Postmortem specimen shows an arachnoid protrusion (asterisk) at the cerebral convexity that penetrates the dura mater (D) through a smooth-contoured single defect (A, arrow) or mesh-like lacunae $(B$, arrow). $C-E$, Schematic drawings of vesicular $(C)$, tubular $(D)$, and extensive types $(E)$ of AP. AM indicates arachnoid membrane; IT, inner table; OT, outer table; SAS, subarachnoid space; V, cortical vein.

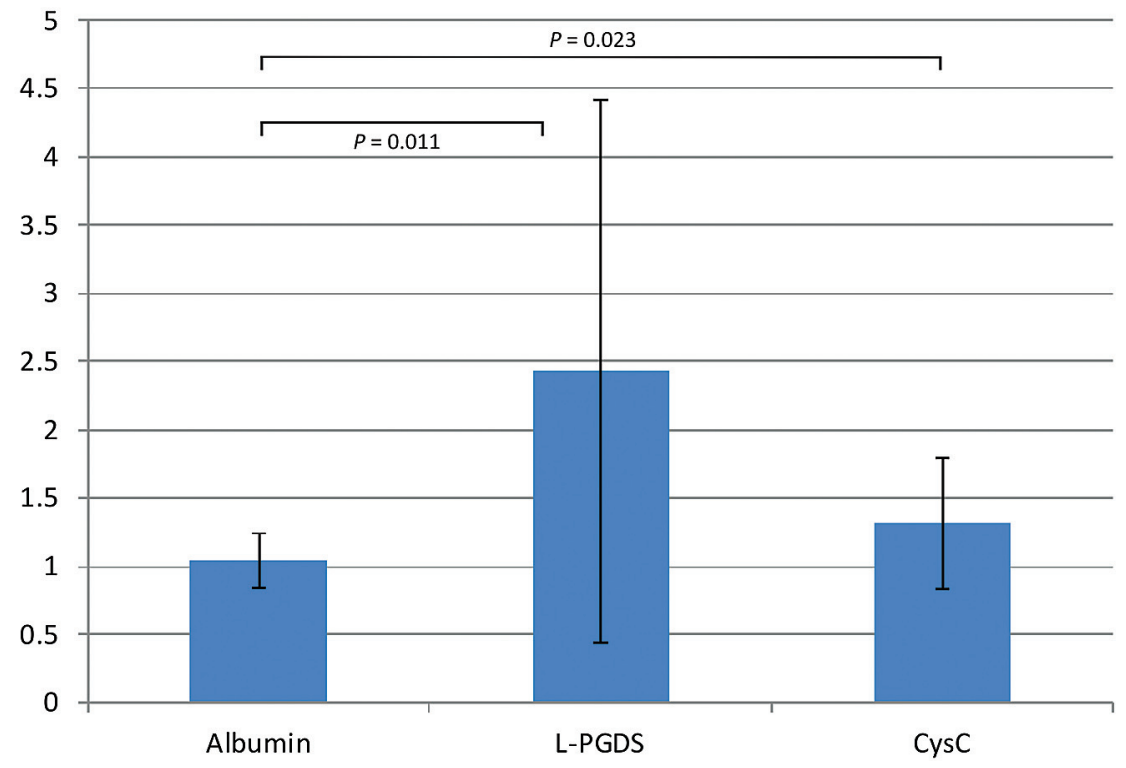

FIG 3. Diploic vein/peripheral vein ratios for PGDS and CysC.

thologies, ${ }^{29,30}$ and sinus pericranii. ${ }^{31}$ The distribution of DVs in the whole cranium is difficult to assess by intraoperative local observation or postmortem dissection because DVs lie intraosseously, surrounded by hard cortical bone. However, high-resolution MR imaging has now made the delineation of these fine structures possible in vivo.
A previous investigation revealed the major pathways of the cranial DVs. ${ }^{19}$ These pathways are distributed extensively throughout the cranium; in fact, at least 1 major route could be interrupted during any standard craniotomy procedure. In our 15 patients, diploic venous blood was collected without difficulty, regardless of the type of craniotomy.

The present study focused on delineating the APs and contiguous DVs throughout the cranium. By a combination of T2weighted sequences and contrast images, APs and contiguous DVs were consistently visualized throughout the cranium with their characteristic appearance dependent on their regional location. Of note, while various types of APs were delineated in the cranial cavity, all the identified APs in the pterional region were the tubular type. In addition, our laboratory investigation of PGDS and CysC levels confirmed that CSF and dissolved, low-molecular-weight proteins can pass through APs into DVs - that is, DVs function not only as venous channels formed in the diploe with abundant mutual connections with the extracranial venous systems ${ }^{19}$ but also as CSF drainage pathways to the extracranial spaces. In the physiologic condition, gadolinium did not permeate the 
blood-brain barrier to dissolve into CSF, ${ }^{32,33}$ while DVs were wellenhanced on contrast examination. This enhancement indicated that gadolinium moved in the arterial blood stream to reach the arterioles and capillaries of the dura mater, next passing through the venules, and finally pouring into DVs. Furthermore, our laboratory results showed that PGDS and CysC could permeate APs and dissolve into DVs in the context of a normal range of intracranial pressure. Therefore, we considered that some quantity of intracranial CSF may drain through APs and DVs (Fig 2C-E).

The next step for these investigations would be the quantification of differences in CSF permeability in each cranial region and differences in CSF permeability in the context of increased intracranial pressure. Because various factors relevant to the proteomics experiments could have influenced the outcome of this study, ${ }^{24}$ it will be important to provide confirmation of the validity and reproducibility of the PGDS and $\mathrm{Cys} C$ results and verification by other appropriate biomarkers. Despite the shortcomings in our methodology, we believe that the present preliminary results convincingly support the notion that at least a fraction of intracranial CSF is drained through APs and DVs.

In the present study, the number of APs identified in the population older than 18 years of age was, except for in the pterional region, more than 3-fold higher compared with number of APs observed in patients younger than 18 years of age. As previously suggested, ${ }^{34}$ the maturation of the dura mater may be related to the development of APs.

\section{CONCLUSIONS}

APs and contiguous DVs are distributed throughout the entire cranium and can be delineated by high-resolution MR imaging. Some portion of the intracranial CSF may be physiologically drained through AP-DV pairs.

\section{REFERENCES}

1. Glimcher SA, Holman DW, Lubow M, et al. Ex vivo model of cerebrospinal fluid outflow across human arachnoid granulations. Invest Ophthalmol Vis Sci 2008;49:4721-28

2. Kapoor KG, Katz SE, Grzybowski DM, et al. Cerebrospinal fluid outflow: an evolving perspective. Brain Res Bull 2008;77:327-34

3. Pollay $M$. The function and structure of the cerebrospinal fluid outflow system. Cerebrospinal Fluid Res 2010;7:9

4. Sakka L, Coll G, Chazal J. Anatomy and physiology of cerebrospinal fluid. Eur Ann Otorhinolaryngol Head Neck Dis 2011;128:309-16

5. Chen F, Deng XF, Liu B, et al. Arachnoid granulations of middle cranial fossa: a population study between cadaveric dissection and in vivo computed tomography examination. Surg Radiol Anat 201 1;33:215-21

6. Farb RI. The dural venous sinuses: normal intraluminal architecture defined on contrast-enhanced MR venography. Neuroradiology 2007;49:727-32

7. Haroun AA, Mahafza WS, Al Najar MS. Arachnoid granulations in the cerebral dural sinuses as demonstrated by contrast-enhanced 3D magnetic resonance venography. Surg Radiol Anat 2007;29:323-28

8. Ikushima I, Korogi $Y$, Makita O, et al. MRI of arachnoid granulations within the dural sinuses using a FLAIR pulse sequence. Br J Radiol 1999;72:1046-51

9. Koshikawa T, Naganawa S, Fukatsu H, et al. Arachnoid granulations on high-resolution MR images and diffusion-weighted MR images: normal appearance and frequency. Radiat Med 2000;18:187-91

10. Leach JL, Meyer K, Jones BV, et al. Large arachnoid granulations involving the dorsal superior sagittal sinus: findings on MR imaging and MR venography. AJNR Am J Neuroradiol 2008;29:1335-39

11. Liang L, Korogi $Y$, Sugahara T, et al. Normal structures in the intracranial dural sinuses: delineation with 3D contrast-enhanced magnetization prepared rapid acquisition gradient-echo imaging sequence. AJNR Am J Neuroradiol 2002;23:1739-46

12. Miabi Z, Midia R, Rohrer SE, et al. Delineation of lateral tentorial sinus with contrast-enhanced MR imaging and its surgical implications. AJNR Am J Neuroradiol 2004;25:1811-18

13. Roche J, Warner D. Arachnoid granulations in the transverse and sigmoid sinuses: CT, MR, and MR angiographic appearance of a normal anatomic variation. AJNR Am J Neuroradiol 1996;17:677-83

14. Trimble CR, Harnsberger HR, Castillo M, et al. "Giant" arachnoid granulations just like CSF?: NOT!! AJNR Am J Neuroradiol 2010;31:1724-28

15. Mase M, Yamada K, Shimazu N, et al. Lipocalin-type prostaglandin D synthase (beta-trace) in cerebrospinal fluid: a useful marker for the diagnosis of normal pressure hydrocephalus. Neurosci Res 2003;47:455-59

16. Haybaeck J, Silye R, Soffer D. Dural arachnoid granulations and "giant" arachnoid granulations. Surg Radiol Anat 2008;30:417-21

17. Yew M, Dubbs B, Tong O, et al. Arachnoid granulations of the temporal bone: a histologic study of dural and osseous penetration. Otol Neurotol 2011;32:602-09

18. Johnston K, Walji AH, Fox RJ, et al. Access to cerebrospinal fluid absorption sites by infusion into vascular channels of the skull diplö. J Neurosurg 2007;107:841-43

19. Tsutsumi S, Nakamura M, Tabuchi T, et al. Calvarial diploic venous channels: an anatomic study using high-resolution magnetic resonance imaging. Surg Radiol Anat 2013;35:935-41

20. Urade Y, Hayashi O. Prostaglandin D synthase: structure and function. Vitam Horm 2000;58:89-120

21. Yamashima T, Sakuda K, Tohma Y, et al. Prostaglandin D synthase (beta-trace) in human arachnoid and meningioma cells: role as a cell marker or in cerebrospinal fluid absorption, tumorigenesis, and calcification process. J Neurosci 1997;17:2376-82

22. Huang YC, Lyu RK, Tseng MY, et al. Decreased intrathecal synthesis of prostaglandin D2 synthase in the cerebrospinal fluid of patients with acute inflammatory demyelinating polyneuropathy. J Neuroimmunol 2009;206:100-05

23. Kanekiyo T, Ban T, Aritake K, et al. Lipocalin-type prostaglandin D synthase/beta-trace is a major amyloid beta-chaperone in human cerebrospinal fluid. Proc Natl Acad Sci U S A 2007;104:6412-17

24. Carrette O, Burkhard PR, Hughes S, et al. Truncated cystatin C in cerebrospinal fluid: technical [corrected] artefact or biological process? Proteomics 2005;5:3060-65

25. García-González U, Cavalcanti DD, Agrawal A, et al. The diploic venous system: surgical anatomy and neurosurgical implications. Neurosurg Focus 2009;27:E2

26. Benndorf $\mathrm{G}$, Lehmann. Bilateral diploic arteriovenous fistula causing scalp hematoma. J Neurosurg 2004;100:950-55

27. Burger IM, Tamargo RJ, Broussard J, et al. Combined surgical and endovascular treatment of a spontaneous diploic arteriovenous fistula: case report. J Neurosurg 2005;103:179-81

28. Shim JH, Yoon SM, Shim JJ, et al. A case of intraosseous dural arteriovenous fistulas involving diploic vein treated with transarterial Onyx embolization. J Korean Neurosurg Soc 2011;50:260-63

29. Ohigashi $Y$, Tanabe A. A huge frontal meningioma associated with intraoperative massive bleeding and severe brain swelling: case report. J Clin Neurosci 2001;8(suppl 1):54-58

30. Tsutsumi S, Yasumoto Y, Ito M. Case of calvarial fibrous dysplasia presenting with cyst degeneration [in Japanese]. No Shinkei Geka 2011;39:163-68

31. Rizvi M, Behari S, Singh RK, et al. Sinus pericranii with unusual features: multiplicity, associated dural venous lakes and venous anomaly, and a lateral location. Acta Neurochir (Wien) 2010;152:2197-204

32. Bozzao A, Floris R, Fasoli F, et al. Cerebrospinal fluid changes after intravenous injection of gadolinium chelate: assessment by FLAIRMR imaging. Eur Radiol 2003;13:592-97

33. Sorensen AG. Science to practice: blood-brain barrier leakage- one size does not fit all. Radiology 2010;257:303-04

34. Squier W, Lindberg E, Mack J, et al. Demonstration of fluid channels in human dura and their relationship to age and intradural bleeding. Childs Nerv Syst 2009;25:925-31 